\title{
On the Unique Factorization Property of the Ring of Nash Functions
}

By

\author{
Masahiro SHIOTA*
}

\section{$\S$ 1. Introduction}

Bochnak and the author showed a necessary and sufficient condition for the ring of analytic functions on a real analytic manifold to be a unique factorization ring [1] and [8]. In this paper we consider the Nash function case. We say that a semi-algebraic set in $\mathbb{R}^{n}$ is a Nash manifold if it is an analytic manifold, and that an analytic function on a Nash manifold is a Nash function if the graph is semi-algebraic. We define a Nash mapping in the same way. The definition of Nash manifold is different to that of Palais [6]. He added moreover the compactness in the conditions, but we don't need the assurnption when we consider the unique factorization property of the Nash function ring. We remark the following fact the compact case of which is a result of Nash [6]. Let $M$ be a $C^{1}$-manifold. $\quad M$ is $C^{1}$-diffeomorphic to a $N a s h$ manifold if and only if $M$ is compact or there exists a compact $C^{1}$-manifold $M^{\prime}$ with boundary such that $M$ is $C^{1}$-diffeomorphic to $M^{\prime}-\partial M^{\prime}$. This follows from [6] and the results in Section 2.

Theorem. The ring of Nash functions on a Nash manifold $M$ is a unique factorization ring if and only if $M$ is connected and satisfies $H^{1}\left(M ; \mathbb{Z}_{2}\right)=0$.

We write this ring as $N(M)$. Bochnak proved essentially the sufficiency and the necessity in the case when $M$ is of dimension 2 [2]. The proof of the necessity in the general case is based on a theorem of Thom about the realization of homology classes [9]. As we shall consider the non-compact case, we need a well imbedding of Nash manifold into a Euclidean space. The imbedded manifold has a Nash tubular neighborhood. That will shows the

Received September 17, 1979.

* Research Institute for Mathematical Sciences, Kyoto University, Kyoto 606, Japan. 
existence of Nash approximation of any $C^{\infty}$-mapping between Nash manifolds.

\section{§2. Well Imbedding of Nash Manifold}

Lemma (Mostowski). Let $X$ be a closed semi-algebraic set in $\boldsymbol{R}^{n}$. Let $Y$ be its complement. Then there exists a continuous function defined on $\boldsymbol{R}^{n}$ such that the restriction on $Y$ is a Nash function and the zero points set is $X$.

The author's correction of the proof of this lemma is quoted in BochnakEfroymson "Real algebraic geometry and the 17th Hilbert problem", Math. Ann., 251 (1980), 213-241.

We remark that the graph of the function in the above lemma is semialgebraic.

Proposition 1. Let $M \subset \boldsymbol{R}^{n}$ be a connected Nash manifold. Then there exist a regular compact algebraic set $A \subset \boldsymbol{R}^{N}$, a closed semi-algebraic subset $B$ of $A$ and a connected component $M^{\prime}$ of $A-B$ such that

1) $M$ is Nash diffeomorphic to $M^{\prime}$,

2) $B$ is locally everywhere normal crossings in $A$, and

3) if $x$ is a point of $B, M^{\prime}$ is connected in an arbitrarily small solid sphere centered at $x$.

Condition 2) means that for every point $\xi \in A$, there exists a local coordinate system $\left(z_{1}, \ldots, z_{N^{\prime}}\right)$ for $A$ centered at $\xi$ such that $B$ is the zero points set of a monomial of those $z_{i}$.

Proof of the proposition. If $M$ is compact, we know already the proposition in [6] Section 14. Hence we consider the non-compact case. Since $\boldsymbol{R}^{n}$ is Nash diffeomorphic to an open half space of the sphere $S^{n}$, we assume that $M$ is contained in $S^{n}$. Hence the closure $\bar{M}$ is compact. By the fundamental properties of semi-algebraic sets, the boundary $\partial M=\bar{M}-M$ is closed semialgebraic, but it is not necessarily algebraic. We want to modify the imbedding of $M$ in $\boldsymbol{R}^{n+1}$ so that $\partial M$ is a point. By the above lemma there exists a continuous function $f$ on $\boldsymbol{R}^{n+1}$ such that the restriction on $\boldsymbol{R}^{n+1}-\partial M$ is a Nash function and that the zero points set of $f$ is $\partial M$. Consider the image of $M$ by the mapping $x=\left(x_{1}, \ldots, x_{n+1}\right) \rightarrow\left(f(x) x_{1}, \ldots, f(x) x_{n+1}\right)$. Then it is Nash diffeomorphic to $M$, and the boundary is $\{0\}$. Therefore we can assume from the beginning that $\partial M$ is a point. Let $K$ be the smallest algebraic set containing $M$. 
Considering in $S^{n+1}$ if necessary, we assume the compactness of $K$. By the normalization of $K$, we reduce the problem to the case when each point of $M$ is a regular point of $K$ (see [6]). We apply the desingularization in [4] to $(K, \partial M)$. Then we have $A, B$ and $M^{\prime}$ in the proposition except for the condition 3).

Now we want to modify $B$ and $M^{\prime}$ to satisfy 3 ). We will find a neighborhood of $B$ and a semi-algebraic set contained in it so that $A$, the semi-algebraic set and the intersection of the exterior of the neighborhood with $M^{\prime}$ are what we wanted. Let $\xi$ be any point of $B$. The condition 2) means the existence of a neighborhood $U$ of $\xi$ in $A$ such that $(U, U \cap B)$ is diffeomorphic to $\left(\mathbb{R}^{m},\left\{z_{1} \cdots z_{l}=0\right\}\right)$ for the coordinate system $\left(z_{1}, \ldots, z_{m}\right)$ of $\mathbb{R}^{m}$. Let $\alpha: \mathbb{R}^{m} \rightarrow \mathbb{R}^{m}$ be defined by

$$
\alpha\left(z_{1}, \ldots, z_{m}\right)=\left(z_{1}^{3}-z_{1}, \ldots, z_{l}^{3}-z_{l}, z_{l+1}, \ldots, z_{m}\right) .
$$

Then $\alpha$ is transversal to the hyper surfaces $\left\{z_{1}=0\right\}, \ldots,\left\{z_{l}=0\right\}$, the restriction on $\left\{\left|z_{i}\right|>1\right.$ for $\left.i=1, \ldots, l\right\}$ is diffeomorphic onto $\left\{z_{i} \neq 0\right.$ for $\left.i=1, \ldots, l\right\}$, and the restriction on $\left\{\left|z_{i}\right|>2 / 3\right.$ for $\left.i=1, \ldots, l\right\}$ is an immersion. Let $\varphi_{U}: U \rightarrow U$ be the mapping invited by $\alpha$. We can easily joint $\varphi_{U}$ and $\varphi_{U^{\prime}}$ for a neighborhood $U^{\prime}$ of another point $\xi^{\prime}$. Thus we have a $C^{\infty}$ mapping $\varphi: A \rightarrow A$ and small neighborhoods $V_{1} \subset V_{2}$ of $B$ such that $\varphi$ is transversal to $B$, that the restriction on the complement of $V_{1}$ is an immersion and that the restriction on $V_{2}^{c}$ is closed to the identity in $C^{\infty}$-topology and diffeomorphic to $A-B$. Let $\psi: A \rightarrow A$ be a Nash approximation of $\varphi$ (see [6]). Then $\psi$ has the same properties as $\varphi$. Hence $\psi^{-1}(B)$ is semi-algebraic and locally everywhere normal crossing in $A$, and the restriction of $\psi$ on a union of connected components of $A-\psi^{-1}(B)$ is Nash diffeomorphic to $A-B$. These show that $A, \psi^{-1}(B)$ and a connected component of $A-\psi^{-1}(B)$ satisfy the conditions 1$), 2$ ) and 3). Thus the proposition is proved.

Remark. We don't know if $B$ in the above proposition is algebraic. By [6], we can approximate a $C^{\infty}$-mapping between compact Nash manifolds by Nash mappings in the $C^{\infty}$-topology. In the same way, using the proposition above we prove this in the compact open $C^{\infty}$-topology without the compact assumption.

The following proposition follows immediately.

Proposition 2. Let $M$ be a non-compact Nash manifold. Then there 
exist Nash manifolds $M_{1} \subset M_{2}$ in a compact regular algebraic set $A \subset \mathbb{R}^{N}$ such that

(1) $M_{1}$ and $M_{2}$ are analytically diffeomorphic and open in $A$,

(2) $M$ is Nash diffeomorphic to $M_{1}$,

(3) the closure $\bar{M}_{2}$ is a Nash manifold with boundary,

(4) a collar of $\partial M_{2}$ in $\bar{M}_{2}$ contains $\partial M_{1}$, and

(5) $\bar{M}_{1} \subset M_{2}$

The problem whether $M_{1}$ and $M_{2}$ in the proposition above are Nash diffeomorphic is open. Moreover, are any two $C^{\infty}$-diffeomorphic Nash manifolds Nash diffeomorphic?

\section{§. Proof of the Theorem}

Proof of the sufficiency. By Proposition $1 M$ has a Nash tubular neighborhood $U$ in a suitable Euclidean space. Let $\mathfrak{P}$ be the ideal of Nash functions on $U$ which vanish on $M$. There is a natural surjective homomorphism from $N(U)$ to $N(M)$ whose kernel is $\supsetneqq$. As $N(U)$ is Noetherian [3], [7], so is $N\left(M_{i}\right)$. If $M$ is connected and satisfies $H^{1}\left(M ; \mathbb{Z}_{2}\right)=0$, then $U$ satisfies the same conditions. Hence, from the result of Bochnak [2] $N(U)$ is a Noetherian unique factorization ring. Consider naturally $N(M)$ as a subring of $N(U)$. Then the sufficiency follows from the next easy lemma.

Lemma. Let $A \supset B$ be Noetherian rings, a be an ideal of $A$ such that $A$ is the direct sum of $B$ and a. If $A$ is a unique factorization ring, so is $B$.

Proof of the necessity. The results of Thom about the realization of homology classes [9] are valid also in the case of manifold with boundary, like the following lemma. The author was pointed out this by Professor Adachi.

Lemma. Let $L$ be an m-dimensional compact $C^{\infty}$-manifold with boundary such that $H^{1}\left(L ; \mathbb{Z}_{2}\right) \neq 0$. Then there exists a compact $C^{\infty}$-submanifold $L^{\prime}$ in L of codimension 1 such that

(1) if $L^{\prime}$ has a boundary, $\partial L^{\prime}$ is contained in $\partial L$,

(2) the fundamental homology class of $L^{\prime}$ in $H_{m-1}\left(L^{\prime} ; \mathbb{Z}_{2}\right)$ is not zero in $H_{m-1}\left(L, \partial L ; \mathbb{Z}_{2}\right)$ (here we consider the relative homology class in place of homology class if $L^{\prime}$ has a boundary), and

(3) $L^{\prime}$ is transversal to $\partial L$. 
Now we prove the necessity. Assume $N(M)$ to be a unique factorization ring. The connectedness of $M$ is trivial. At first we assume that $M$ is compact and of dimension $m$ and satisfies $H^{1}\left(M ; \mathbb{Z}_{2}\right) \neq 0$. From the realization of homology classes we have a one codimensional, compact, connected and $C^{\infty}$ submanifold $M^{\prime}$ in $M$ such that $\left[M^{\prime}\right] \neq 0$ in $H_{m-1}\left(M ; \mathbb{Z}_{2}\right)$. It follows that $M-M^{\prime}$ is connected. Lei $f$ be a $C^{\infty}$-function defined on $M$ such that $f^{-1}(0)$ $=M^{\prime}, f \geqq 0$ and for any point $x$ of $M^{\prime}, f$ is the square of a regular $C^{\infty}$-function round $x$. We will approximate $M^{\prime}$ by a suitable zero points set of Nash functions. We can prove this more easily. But the author shall need a strong form in another paper. Let $G_{n, k}$ be the Grassmanian manifold, $\ddot{\xi}_{n, k}$ the naturally defined vector bundle over $G_{n, k}$ with fibre $\mathbb{R}^{k}$. Here $n$ is the dimension of the Euclidean space containing $M$. Then these are Nash manifolds and the projection $\xi_{n, h} \rightarrow G_{n, k}$ is a Nash mapping. Define a Nash mapping $\pi_{1}: M \rightarrow G_{n, m}$ by

$$
\pi_{1}(x)=T_{x} M \quad \text { for } x \text { in } M .
$$

Let $U$ be a Nash tubular neighborhood of $M$ whose closure is compact. We can naturally extend $\pi_{1}$ on $U$. We use the same $\pi_{1}$ for the extension. $\pi_{1}$ is also a Nash mapping. Let $\pi_{2}: M^{\prime} \rightarrow G_{n, 1}$ be a $C^{\infty}$-mapping defined by

$$
\pi_{2}(x) \subset \pi_{1}(x) \text { and } \pi_{2}(x) \perp T_{x} M^{\prime} \quad \text { for } x \text { in } M^{\prime} .
$$

If we take $n$ larger, then we can extend $\pi_{2}$ on $\mathbb{R}^{n}$. Let $\pi_{3}$ be a Nash approximation of the extension such that they are sufficiently close on $\bar{U}$ in the $C^{0}$ topology. Let $\pi_{1}^{\prime}: U \rightarrow G_{n, m-n}$ and $\pi_{3}^{\prime}: U \rightarrow G_{n, n-1}$ be the Nash mapping such that

$$
\pi_{1}(x) \perp \pi_{1}^{\prime}(x) \text { and } \pi_{3}(x) \perp \pi_{3}^{\prime}(x) \text { for any } x \text { in } U .
$$

For each $i=1, \ldots, n$, we write as $X_{i}$ the vector field $\partial / \partial x_{i}$ on $U$ where $\left(x_{1}, \ldots, x_{n}\right)$ is the coordinate system of $\mathbb{R}^{n}$. Then there are vector fields $X_{i}^{1}, X_{i}^{2}, i=1, \ldots, n$, on $U$ such that

$$
\begin{aligned}
& X_{i}=X_{i}^{1}+X_{i}^{2} \\
& X_{i, x}^{1} \subset \pi_{3}(x) \text { and } X_{i x}^{2} \subset \pi_{3}^{\prime}(x) \text { for any } x \text { in } U .
\end{aligned}
$$

We call $X_{i}^{1}$ as the $\pi_{3}$-component. It is easy to see that $X_{i}^{j}$ are Nash mappings from $U$ to $T U$. Let $Y_{i}$ be the $\pi_{1}$-component of $X_{i}^{1}$ with respect to $\pi_{1}$ and $\pi_{1}^{\prime}$, and $Z_{i}$ be the restriction of $Y_{i}$ on $M$. Then $Z_{i}$ are Nash mapping from $M$ to $T M$. It follows moreover that for any point $x$ in $M^{\prime}$ there exists $i$ such that $Z_{i x} \neq 0$ and $Z_{j}=Z_{i} \times($ a Nash function) around $x$ for each $j=1, \ldots, n$. This means that 
at least one of $f_{i}=Z_{i} f$ is regular at each point of $M^{\prime}$ and $M^{\prime}$ is a connected component of $V\left(f_{1}, \ldots, f_{n}\right)$ the zero points set of $f_{1}^{2}+\cdots+f_{n}^{2}(*)$. Let $g$ be a Nash approximation of $f$ on $M$ in the $C^{2}$-topology. We put $g_{i}=Z_{i} g$. From the properties of $f$ and $Z_{i},(*)$ remains valid after the approximation, that is, at least one of $g_{i}$ is regular at each point of $V_{1}$ the connected component of $V\left(g_{1}, \ldots, g_{n}\right)$ which is close by $M^{\prime}(* *)$. Therefore the pairs $\left(M, M^{\prime}\right)$ and $\left(M, V_{1}\right)$ are diffeomorphic. We consider a prime ideal $\mathfrak{B}=\left\{f \in N(M)|f|_{V_{1}} \equiv 0\right\}$. By the fact that $N(M)$ is of Krull dimension $m$, we see easily the height of $\mathfrak{B}$ is one. From the assumption of the unique factoriality $\mathfrak{P}$ is a principal ideal, that is, there is a Nash function $h$ on $M$ that generates $\mathfrak{P}$. The property (**) means that $h$ is regular on $V_{1}$ and that $V_{1}$ is a connected component of $V(h)$. Moreover we know in [5] that $V(h)$ is connected and then equal to $V_{1}$. Hence we have

$$
\begin{gathered}
h^{-1}\left(\boldsymbol{R}_{+}\right) \neq \phi, \quad h^{-1}\left(\boldsymbol{R}_{-}\right) \neq \phi, \quad M-V_{1}=h^{-1}\left(\boldsymbol{R}_{+}\right) \cup h^{-1}\left(\boldsymbol{R}_{-}\right) \\
\text {where } \quad \boldsymbol{R}_{+}=\{t \supsetneqq 0\}, \quad \boldsymbol{R}_{-}=\{t \supsetneqq 0\} .
\end{gathered}
$$

This contradicts the connectedness of $M-V_{1}$. Thus we proved the compact case of $M$.

It remains to prove the non-compact case. We assume that $H^{1}\left(M ; Z_{2}\right) \neq 0$ and $M$ is connected. We use the same notation as in Proposition 2. By the realization lemma above we can find Nash functions $h_{1}, \ldots, h_{k}$ on $M_{2}$ in the same way as above such that

1) $\quad M_{2}-V$ is connected and $V$ is not empty where $V=V\left(h_{1}, \ldots, h_{k}\right)$,

2) at least one $h_{i}$ is regular at each point of $V$, and

3) $\bar{V}$ is the product $\partial V \times[0,1)$ in a collar $W$ of $\partial M_{2}$ in $\bar{M}_{2}$ where $W$ contains $\partial M_{1}$.

Let $\bar{h}_{i}$ be the restrictions of $h_{i}$ on $M_{1}$ for $i=1, \ldots, k, V_{1}$ be the connected component of $V \cap M_{1}$ which contains $V-W . \quad$ By $\mathfrak{B}$ we denote the prime ideal of Nash functions on $M_{1}$ which vanish on $V_{1}$. Then the height of $\mathfrak{P}$ is one and the common zero points set of $\mathfrak{P}$ is $V_{1}$. The conditions 1), 2) and 3) means that $V_{1}-W$ is not empty and $M_{1}-\left(V_{1} \cup W\right)$ is connected. Hence for any two points $a_{1}, a_{2}$ in $M_{1}-\left(V_{1} \cup W\right)$, there is a continuous path from $a_{1}$ to $a_{2}$ in $M_{1}-\left(V_{1}\right.$ $\cup W$ ). If $\mathfrak{P}$ is generated by a function, then the restriction of the function on the path is a positive or negative function. This contradicts the condition 2). Hence $\mathfrak{P}$ is of height one but not a principal ideal. Therefore $N\left(M_{1}\right)$ is not a unique factorization ring. This completes the proof of the theorem. 


\section{References}

[1] Bochnak, J., Sur la factorialité des anneaux de fonctions analytiques, C.R. Acad. Sci. Paris, 279 (1974), 269-272.

[2] —— Sur la factorialité des anneaux de fonctions de Nash, Comm. Math. Helv., 52 (1977), 211-218

[3] Efroymson, G. A., A nullstellensatz for Nash rings, Pacific J. of Math., 54 (1974), $101-112$

[4] Hironaka, H., Resolution of singularities of an algebraic variety, I-II, Ann. Math., 79 (1964), 109-326.

[5] Mostowski, T., Some properties of the ring of Nash functions, Am. Scuola Norm. Sup. Pisa, III, 2 (1976), 245-266.

[6] Palais, R., Equivariant real algebraic differential topology, Part I, Smoothness categories and Nash manifolds, Notes Brandies Univ., 1972.

[7] Risler, J. J., Sur l'anneau des fonctions de Nash globales, C.R. Acad. Sci. Paris, 276 (1973), 1513-1516.

[ 8 ] Shiota, M., Sur la factorialité de l'anneau des fonctions analytiques, C. R. Acad. Sci. Paris, 285 (1977), 253-255.

[9] Thom, R., Quelques proprietés globales des variétés différentiables, Comm. Math. Helv., 28 (1954), 17-86. 
